\title{
The effect of ready-to-eat cereal consumption on energy intake, body weight and anthropometric measurements
}

\author{
S. L. Hull, A. V. Matthews, R. Re, F. Angus and K. L. Johnston \\ Nutrition Research Section, Leatherhead Food Research, Randalls Road, Leatherhead, Surrey KT22 7RY, UK
}

\begin{abstract}
Although many factors contribute to overweight and obesity, snacking is suggested to be one source of excess energy intake, which contributes to an undesirable weight profile. While snacking patterns among children are well studied, the actual relationship between snacking and changes in adult body weight remains controversial, since little data have been published on eating patterns and frequency among this group ${ }^{(1)}$ and both longitudinal and randomised controlled intervention trials in this area are scarce. Indeed, snacking has been shown to be one source of excess energy intake that is more common in those individuals seeking weight loss treatment and which may contribute to an undesirable weight profile. As such, the purpose of this study was to determine the effects of consumption of a structured post-dinner snack in the form of ready-to-eat cereal (RTEC) in place of a normal evening snack on body weight, anthropometric measurements and energy intake in overweight habitual evening snackers.

The study was a randomised, controlled, parallel intervention trial performed in overweight, self-reported habitual evening snackers. Ethical approval was obtained from West Kent Research Ethics Committee (reference no. 08/H1103/71). Seventy participants (18 male and 52 female) (mean (SD); age, $40.4(9.0)$ years, BMI, $29(3.3) \mathrm{kg} / \mathrm{m}^{2}$ ) were recruited and randomly assigned to either the control (NC) or cereal (CR) group. Over a period of 6 weeks, the CR group was instructed to consume a serving of RTEC ( $>25 \mathrm{~g}$ but $<45 \mathrm{~g}$ ) with $125 \mathrm{ml}$ semi-skimmed milk in place of their normal evening snack. They were provided with scales to weigh the cereal they consumed and a compliance sheet to record amounts and time of consumption each evening. The NC group did not receive any intervention and were instructed to maintain their usual dietary and exercise habits as closely as possible. Body weight and anthropometric measurements (body fat, waist and hip circumference) were taken on weeks $0,2,4$ and 6 and 3-d diet diaries were completed during the week prior to each of these visits.

A two-factor, mixed between-within repeated measures ANOVA (RM-ANOVA), where diet was the between factor and time was the within factor that was used to analyse the differences in the means across visits between the control and treatment groups. Significant interactions were further analysed using Duncan's multiple range post hoc test to determine where the differences lay and $P$ values $<0.05$ were considered significant.

There were no significant differences between groups in any anthropometric measurements although within the CR group body weight $(P=0.030)$ and waist circumference $(P=0.0003)$ were reduced after the 6 weeks compared with baseline. Food diary analysis showed that there was a clear trend $(P<0.10)$ towards higher daily energy intake in the NC group compared with the CR group and breakdown by macronutrient showed that there was a statistically significant difference between study groups for daily carbohydrate intake with higher amounts consumed on average in the NC group $v$. the CR group $(P=0.031)$. There was also a difference in evening energy intake between the $\mathrm{CR}$ and NC groups, with consumption being lower in $\mathrm{CR}$ compared to NC groups $(P=0.007)$.

Previous work involving supplying cereal for both breakfast and lunch has shown significant weight loss after 2 weeks as a result of reduced energy intake ${ }^{(2)}$ and another study has shown that snacking patterns significantly affect energy and nutrient intakes ${ }^{(3)}$. Based on these findings and those of Waller et al. ${ }^{(4)}$ who conducted a similar study over 4 weeks and showed an attenuation of evening energetic intake, it is reasonable to expect that RTEC consumption as a structured post-dinner snack may result in a decrease in energy intake, with subsequent effects on weight loss and anthropometry. To conclude, the replacement of an evening snack with a structured post-dinner RTEC snack in overweight habitual evening snackers significantly reduces post-dinner energy intake and may contribute to weight management in those individuals seeking to lose weight in the longer term.
\end{abstract}

1. Gatenby SJ (1997) Br J Nutr 77, Suppl., S7-S20.

2. Lightowler HJ \& Henry CJK (2009) Nutr Bull 34, 48-53.

3. Hampl JS, Heaton CL \& Taylor CA (2003) J Hum Nutr Diet 16, 3-11.

4. Waller SM, Vander Wal JS, Klurfeld DM et al. (2004) J Am Coll Nutr 23, 316-321. 